\title{
Prácticas de crianza y desarrollo afectivo y emocional de los niños
}

Parenting styles and affective and emotional development on children

\author{
Martha Frías Armenta \\ Blanca Fraijo Sing \\ César Tapia Fonllem
}

\begin{abstract}
Resumen
La familia es el lugar donde los niños gozan de sus primeras interacciones con el mundo, los padres les proporcionan el soporte emocional y social, además de las actividades de aprendizaje. Las madres, especialmente, invierten grandes cantidades de recursos, tiempo y energía para que puedan sobrevivir y convertirse en adultos exitosos. Teóricamente, las madres y los padres buscan invertir en la crianza de sus hijos, para que éstos logren un proceso de adaptación y sobrevivencia al ambiente que les tocó vivir, por lo tanto, buscan las mejores técnicas para lograrlo. En este sentido, el presente estudio investiga cómo los padres y las madres se perciben a sí mismos en sus prácticas de crianza y en las formas de mostrar afecto a sus hijos e hijas. La muestra la conformaron 201 padres y madres de familia escogidos al azar en la ciudad de Hermosillo, Sonora. Las entrevistas se llevaron a cabo en sus hogares por psicólogas. Estadísticas descriptivas, t de student y chi cuadrada fueron utilizadas para el análisis de datos. Los resultados nos indican que los padres se aprecian a sí mismos como buenos, responsables y amorosos y no utilizaron el castigo físico. Se puede concluir que ellos tienen una percepción positiva de sus prácticas de crianza y de cómo educan a sus hijos.
\end{abstract}

\section{Abstract}

Family is the place where children enjoy their first interactions with the world. Parents provide children emotional, social as well as learning support. Mothers in particular, invest substantial time and energy on their children so they can survive and grow to be successful adults. In theory, mothers and fathers seek to invest in the upbringing of their children in order for them to achieve a process of adaptation and survival in the environment in which they live; therefore, they look for the best tools to achieve this goal. In this sense, the aim of this study is to investigate the perception of mothers and fathers about their parenting styles. The sample was 201 mothers and fathers of Hermosillo, Sonora, Mexico, a northwestern city of Mexico. Students of psychology interviewed the parents in their houses. Descriptive statistics, t student and chi square were used for data analysis. Results indicate that parents identify themselves as good, responsible, loving and they do not report the use of physical punishment. We can conclude that parents have a positive perception of their parenting and educational styles.

Palabras Clave: percepción, prácticas crianza, afecto, educación, adolescentes.

Keywords: perception, parenting styles, adolescents, education, affect. 


\section{Introducción}

La familia es considerada una de las influencias más significativas para el desarrollo de los niños, mientras que para los infantes es una cuestión de sobrevivencia; nada es más crítico para el éxito de los niños que sus padres. Los padres y las madres, especialmente las madres, invierten grandes cantidades de tiempo, recursos, energía y esfuerzo en la crianza de los hijos. Esto les ayuda a los niños a obtener una mejor adaptación al ambiente; entre más inversión paterna tendrán un mejor ajuste. Las variaciones en los tiempos y recursos de la crianza de los padres contribuyen diferencialmente al desarrollo psicológico de los niños, sin embargo, es reconocido que existen factores extra familiares que igualmente influyen (Collins, Maccoby, Steinberg, Hetherington, \& Bornstein, 2000). Los padres son los primeros y más importantes maestros de los niños, ellos les promueven aprendizaje y desarrollo al estructurar múltiples aspectos del ambiente incluyendo: (a) participación en actividades de alfabetismo, (b) actividades de apoyo, (c) y proveyéndolos de materiales de aprendizaje apropiados para su edad (Rodríguez et al., 2009).

Existe una gran variedad de formas sobre cómo interactúan las madres con los niños, dependiendo de los factores individuales, culturales y situacionales que las rodean. Las prácticas incluyen creencias, sentimientos, emociones y cogniciones que se conjugan en las relaciones de las madres y padres con los niños. Cualquier variación en los patrones de crianza de las madres se realiza para asegurar la sobrevivencia, la adaptación y el desarrollo saludable de los niños. Las prácticas de crianza pueden ser entendidas en términos de los roles que los padres juegan con relación a la vida de sus hijos, o en términos de los objetivos de socialización de las prácticas (Hoff, Laursen, \& Tardif, 2002).

Baumrind (1983) propuso una conceptualización de los estilos educativos paternos basado en el tipo de control ejercido por los padres hacia los hijos; de este análisis resultaron tres tipos cualitativamente diferentes: los padres autoritarios, los permisivos y los autoritativos. Los padres autoritarios constituyen padres altos en demandas y bajos en responsividad, los padres pasivos son padres bajos en demandas y altos en responsividad, o bien, como señalan Maccoby y Martin (1983), pueden ser bajos en demandas y bajos en responsividad y, por último, los padres autoritativos son padres altos en demandas y altos en responsividad.

Las prácticas de crianza involucran una relación que se extiende durante toda la vida, ejerciendo influencias mutuas entre padres e hijos en este tiempo (Townsend, 2000). La crianza de los niños es una función compleja en donde intervienen múltiples factores incluyendo la propia historia de los padres, la personalidad y las características de los niños y padres (Belsky, Putnam, \& Crnic, 1997). El modelo Le Vine's sugiere que aquello que los padres pretenden para sus hijos depende de un sistema jerárquico de objetivos, iniciando con el aseguramiento de sobrevivencia básica hasta la realización y obtención de relevantes valores culturales, sociales y económicos (LeVine et al., 1994). Investigaciones en diversos ambientes sugieren que la relación entre contexto sociocultural y los objetivos en las prácticas de crianza es compleja (Harkness, 2002). Los contextos de la vida diaria no sólo definen los parámetros de experiencia, también simbolizan significados culturales importantes. Las comunidades culturales proveen un número posible de contextos, desde los tipos de casas hasta las expectativas de las actividades para los padres y los hijos en diferentes edades y sexos. Los padres escogen, de esa cantidad de posibilidades, los ambientes apropiados para el desarrollo de los niños. El proceso de selección va a depender de las necesidades cambiantes de los padres, de los niños y los contextos. Lo que los adultos hacen en su rol de padres ha ido cambiando dependiendo del desarrollo social, tecnológico, y económico. Existe, Además, una gran diversidad en las actividades que los padres realizan en su rol y cómo lo hacen a través 
de las culturas. Sin embargo, el objetivo de la crianza es el mismo a pesar de las diferencias, los padres deberán hacer lo necesario para que sus hijos se conviertan en adultos competentes que puedan funcionar en la sociedad (Bradley, 2002). Bradley (2002), basándose en esta definición, identificó 5 funciones principales para los padres y las madres: a) sostenimiento, b) estimulación, c) soporte, d) estructura y e) supervisión.

El sostenimiento se refiere a los actos y las condiciones que son asignados para promover la integridad biológica. Los padres deberán de proveer los nutrientes, albergue, y las condiciones para mantener la salud, asegurar la sobrevivencia y los niveles de integridad biológica necesaria para el desarrollo físico y psicológico. La estimulación está relacionada con los datos sensoriales otorgados a los niños, para ayudarlos en su desarrollo psicológico, social, psicomotor y cognitivo. Existen pocos datos acerca de la frecuencia con que los niños están expuestos a objetos, eventos y experiencias, y mucho menos información acerca de cómo ésta varía dependiendo del contexto ambiental (Bradley, 2002).

Bradley, Corwyn, McAdoo, y García Coll (2001) examinaron la frecuencia con la que los niños eran expuestos a objetos comunes y servicios en el ambiente de la casa, utilizando datos de la Encuesta Longitudinal Nacional de Jóvenes (National Longitudinal Survey of Youth) de Estados Unidos de Norte América y encontraron que los infantes americanos-europeos que no se encontraban en pobreza tenían al menos 10 libros, un $67 \%$ de los padres les leían al menos 3 veces a la semana y un tercio de los niños tenían juguetes para empujar y jalar. Una vez que entraron a edad escolar, los padres redujeron la lectura semanal. Las familias de ascendencia europea y asiática con ingresos arriba de la pobreza tenían, también, instrumentos musicales para los niños de primaria. Los resultados de la encuesta mostraron diferencias entre los niños pobres y no pobres con relación a la exposición de objetos y eventos estimulantes en la casa, inclusive en los esfuerzos directos de enseñarles competencias escolares relevantes. Estas diferencias pudieran deberse al conocimiento de los padres, a las creencias, al estrés y al acceso limitado de recursos (Bradley, 2002). Sin embargo, a pesar del menor desempeño de los niños de familias de bajos recursos, existe una gran variabilidad en el lenguaje y las competencias cognitivas (Pan, Rowe, Singer, \& Snow, 2005).

El soporte comprende las respuestas que se proporcionan a las necesidades sociales y emocionales (Bradley, 2002). Los padres asisten a los hijos en enlistar y modular las propiedades motivacionales de las emociones para asegurar el ajuste óptimo con las demandas ambientales. El desarrollo social y emocional de los niños depende de tener un ambiente que responda a las necesidades sociales y emocionales. Existe evidencia según la cual el desarrollo es mejor en ambientes responsivos, es decir, los requerimientos de asistencia del niño son proporcionados a tiempo y de manera predecible y satisfactoria (Bradley, 2002). Rohner (1986) obtuvo evidencia de varios estudios cros-culturales y mostró que las relaciones honestas, amorosas y compasivas promueven un buen ajuste en el sentido de mostrar un buen comportamiento y salud. Las prácticas de crianza inconsistentes y hostiles producen conductas socialmente incompetentes (agresión) en los niños. Los padres que utilizan métodos aversivos para controlar la conducta de los niños los llevan a un funcionamiento sub-óptimo y a relacionarse con compañeros antisociales (Dishion, \& McMahon, 1998).

La estructura está relacionada con la configuración o los arreglos que los padres elaboran para que los niños puedan recibir la estimulación, el soporte y el sostenimiento necesario para su adaptación y sobrevivencia en el ambiente que viven (Wachs, 2000). Una buena estrategia de crianza consiste no sólo en asegurar que la suficiente cantidad de estimulación, 
soporte y sostenimiento sean otorgadas a los niños, sino también en la configuración o estructuración que los niños reciban para alcanzar el ajuste (Bradley, 2002). Un ejemplo que requiere de la estructura parental es el aprendizaje. Existe evidencia que el número de objetos que las personas pueden manejar se incrementa con la edad durante la infancia y que el aprendizaje es fácil con la ausencia de distractores. El desarrollo cognitivo de los niños es afinado no únicamente por el tipo y la cantidad de estímulos que los niños reciben, sino también por la forma como los padres organizan física y temporalmente el ambiente. Las condiciones de aglomeración en las casas dificultan a los niños organizar las cosas para ellos mismos y maximizar la interacción de las personas y los objetos en el ambiente; estas circunstancias limitan la explotación e impiden el juego sofisticado y, en términos prolongados, limitan el éxito y motivan la agresividad (Evans et al.,1991). Adicionalmente, la investigación ha demostrado que los padres tienden a supervisar menos a sus hijos en estas situaciones (Bradley, Corwyn, Caldwell et al., 2000). La estructura también es importante para el desarrollo socio-emocional; cuando el ambiente se percibe incontrolable genera ansiedad y esto incita conducta desadaptada. Existe evidencia dentro de la psicología ambiental sobre cómo los arreglos físicos en las casas, guarderías, y escuelas afectan la probabilidad de que la gente realice ciertas actividades.

A nivel teórico, se argumenta que las prácticas de crianza dependen de las creencias, objetivos y valores que comparten los padres sobre el desarrollo del niño. No obstante, la revisión de la literatura muestra que las prácticas de crianza pueden estar igualmente influenciadas por factores contextuales. De esta manera, algunos investigadores argumentan que las expectativas de las madres y padres acerca de los hijos varían dependiendo de su estatus socioeconómico (SES). Los padres de diferentes SES esperan diferentes tiempos en el desarrollo de los niños. A través de las cul- turas, las madres con SES más alto esperan un desarrollo más temprano en los niños y sus expectativas son más altas (Hoff, Laursen, \& Tardif, 2002). Los estilos parentales igualmente varían dependiendo del SES. Los padres de clase media tienden a ser mas democráticos y los de clase baja más autoritarios (Dornbusch, Ritter, Leiderman, \& Roberts, 1987).

Cole y Tamang (1998) condujeron un estudio con niños en Chhetri-Brahmin y en Tamang Nepal y encontraron diferencias en las prácticas de socialización de las madres. Las madres de Chhetri-Brahmin enseñaron a los niños a mostrar sus emociones y las madres de Tamang pensaban que ellos mismos las podían aprender. Esto está reflejado en el entendimiento de los niños sobre el manejo de las emociones. Los autores comentan que estas prácticas estaban de acuerdo con las creencias religiosas que ellos profesaban (el Budismo Tibetano versus el Hindú).

El estudio de Gaskinss (1995) en las comunidades Mayas de México muestra que las creencias de los padres acerca del desarrollo de los niños contrastan con las de los padres de clase media de Estados Unidos. Los mayas piensan que el desarrollo es generado internamente, y la idea de estimular a los niños mediante el juego no es inculcada. Los patrones de los niños con relación al juego exploratorio parecen estar de acuerdo con esta idea. Los datos anteriores indican cómo las ideas van progresando del sistema de creencias cultural, a las creencias de los padres sobre la socialización de los niños, y las ideas de los niños (Harkness, 2002).

La exposición al lenguaje de los padres está relacionada con el vocabulario que manejan los niños (Nord, Lennon, Liu, \& Chandler, 2000). La participación en actividades de alfabetismo (que les lean libros, les cuenten historias, reciten rimas, números, el alfabeto, o los lleven a museos, etc.) es fundamental para el desarrollo del lenguaje de los niños y el alfabetismo emergente (Raikes et al., 2006). El ambiente físico en la casa también afecta a los niños. Wachs 
(2000) acumuló evidencia en donde se muestra que los niveles de ruido en el hogar estaban negativamente relacionados al desarrollo cognitivo de los niños. Evans, Maxwell, y Hart (1999) encontraron que los padres que viven en casas aglomeradas hablan menos oraciones complejas y tienen menos respuestas verbales con sus hijos comparados con los padres en ambientes menos abarrotados.

Las diferencias individuales en las prácticas de crianza afectan la personalidad y el desarroIlo intelectual de los hijos (Belsky, 2000); no obstante, es importante reconocer qué tan crítica pudiera ser esa influencia en el desarrollo cognitivo, afectivo y social de los niños. Desde el punto de vista de la psicología evolucionista, algunos investigadores proponen que las diferencias ordinarias entre las familias tendrían poco efecto en el desarrollo de los niños, éste sería notorio si la familia estuviera fuera de lo normal (Bjorklund, Yunger, \& Pellegrini, 2002). Los distintos estilos parentales crean diversos climas emocionales en la casa pueden tener un efecto diferencial en el desarrollo de los niños. Carlo, Mestre, Samper, Tur y Armenta (2010) realizaron una investigación longitudinal en 700 niños de entre 10 y 12 años, encontraron que el afecto o soporte de los padres y el razonamiento pro-social fueron predictivos de la conducta pro-social de los jóvenes.

En el mismo sentido, los estilos de crianza de los padres predicen la inteligencia emocional de los niños; la responsividad parental, demandas positivas de los padres y entrenamiento emocional de los mismos están relacionados con un alto desarrollo de inteligencia emocional, mientras que las demandas negativas están relacionadas con una baja (Alegre, 2011). La responsividad parental está asociada con los resultados positivos en el desarrollo de los niños tales como alta auto-regulación y baja conducta externa (Eiden, Edwards, \& Leonard, 2007), alta auto-estima y un mejor ajuste psicológico (Khaleque, Rohner, \& Riaz, 2007). La responsividad parental está relacionada con las prácticas parentales de monitoreo y supervisión, control conductual, concesión de autonomía, demandas y expectativas maduras de los padres, además de la disciplina inductiva (De Clercq, Van Leeuwen, De Fruyt, Van Hiel, \& Mervielde, 2008; Sanders, 2008). Dichas prácticas parentales están relacionadas con el consumo de alcohol (Mogro-Wilson, 2008), alto funcionamiento académico (Wang, Pomerantz, \& Chen, 2007), y menos conducta sexual de riesgo (Baptiste, Tolou-Shams, Miller, Mcbride, \& Paikoff, 2007), alta satisfacción por la vida (Suldo \& Huebner, 2004), alta conducta prosocial y confidencia (Collins \& Barber, 2005). Un estudio longitudinal llevado a cabo con una muestra de 1049 adolescentes midió la correspondencia entre estilos parentales y trayectorias de desarrollo a través de la niñez y la adolescencia. Los resultados mostraron que los estilos parentales fueron diferencialmente relacionados con el consumo de alcohol y cigarro, conducta antisocial y síntomas internalizados mientras que el estilo autoritativo fue asociado al desarrollo óptimo (Luyckx, Tildesley, Soenens, Andrews, Hampson, Peterson, \& Duriez, 2011).

Las demandas negativas de los padres incluye el control psicológico y disciplina punitiva e inconsistente, características que han sido relacionadas con problemas de internacionalización y externalización, bajo bienestar emocional, desórdenes de personalidad, baja conducta pro social, y ansiedad cognitiva (Johnson, Cohen, Chen, Kasen, \& Brook, 2006; Knafo \& Plomin, 2006; Lengua, 2006; Van Leeuwen \& Vermulst, 2004), bajo entendimiento y regulación emocional (Morris, Silk, Steinberg, Myers, \& Robinson, 2007).

Los contextos sociales y físicos donde las familias viven constituyen los nichos de desarrollo de los niños (Bradley, 2002) encargados de regular su microambiente. Los padres y los niños son constructores activos de sus ambientes y también responden a ellos (Wachs, 2000). El proceso de crianza de los niños involucra nu- 
merosos intercambios de momento a momento con el contexto y el niño. De esta manera, se ha encontrado que las interacciones padres e hijos tienen un efecto bidireccional; la personalidad de los niños es moderadora de los estilos parentales de los padres (Meunier, Roskam, \& Browne, 2010). Son pocos los estudios que han examinado esta relación, sin embargo, se ha encontrado que la disciplina inconsistente, conflictiva y severa, así como la carencia de soporte hacia los niños están asociadas con el aumento de los síntomas de externalización (Huh, Tristan, Wade, \& Stice, 2006). Igualmente, Larsson (2008) encontró una relación recíproca entre estilos parentales inefectivos y los síntomas de externalización. Sin embargo, no existe suficiente investigación para determinar quién ejerce mayor influencia en esa relación.

La literatura nos indica que los estilos de crianza de los padres ejercen un efecto en la conducta de los niños y adolescentes. Los padres y madres invierten en la crianza de los hijos e hijas para que ellos puedan tener un mejor proceso de adaptación y sean adultos exitosos. Se ha investigado sobre el efecto de los estilos de crianza en la conducta de los hijos e hijas, pero no existe investigación abundante sobre la percepción que tienen los padres sobre sus estilos. Independientemente de la conducta, es importante conocer qué es lo que piensan de ellos mismos. Por lo tanto, el objetivo del presente estudio es analizar la percepción de los padres sobre los estilos parentales, las muestras de afecto y las formas de educar y disciplinar a sus hijos.

\section{Método}

\section{Sujetos}

La muestra quedó integrada por 201 padres de familia de clase media de la ciudad de Hermosillo, Sonora. La edad promedio fue de 42 años, con una desviación estándar de 8.14. El $50 \%$ fueron mujeres y $50 \%$ fueron varones, $\mathrm{El}$ número de hijos que tuvieron oscilaba entre 1 y 12 con una media de 3.07 (D.S. 1.5).

\section{Instrumentos}

El instrumento fue elaborado por un grupo de investigadores de universidades que pertenecen a CUMex. Las primeras preguntas correspondían a cuestiones demográficas como edad, grado escolar, sexo, y número de hijos. El resto concernió a la percepción de los padres acerca de las prácticas de crianza de ellos y otros padres; en 10 preguntas abiertas, los padres expresaban su apreciación acerca de ellos mismos, las formas de educar, la demostración de afecto y la forma de corregir a sus hijos. Los datos fueron codificados con base en la teoría de Baumrind (1983) sobre la crianza de los hijos, estilos autoritarios, autoritativos, y permisivos, según la definición dada en el marco teórico. Las formas de demostrar afecto fueron codificadas de acuerdo con la teoría de Bradley (2002). Las muestras verbales de afecto (decir que los quieren) fueron catalogadas como cariño verbal y las muestras físicas (abrazos, besos, etc.) como instrumental. Las formas de disciplinar a los hijos fueron codificadas de acuerdo con la instrumentación propuesta por Straus (1999); el castigo verbal es cualquier agresión verbal y el castigo físico son daños físicos como golpes, quemaduras, escoriaciones, etcétera.

\section{Procedimiento}

Los padres de familia fueron visitados en sus casas. Pasantes de Psicología administraron los cuestionarios en alrededor de 10 minutos. Los pasantes explicaron los objetivos de la investigación, resolvieron dudas, aseguraron la confidencialidad de la información y pidieron el consentimiento de los padres para participar, se les indicó que en cualquier momento podían detener el llenado del instrumento si algo no les parecía. 


\section{Análisis de datos}

Los datos fueron, en principio, codificados y analizados con estadísticas univariadas, medias y desviaciones estándar para las variables continuas y frecuencias para las variables categóricas. Posteriormente, se realizaron $t$ test para ver diferencias de medias en la percepción de los padres sobre la forma de educar, tanto de propia como de otros padres y sobre la demostración de afecto y disciplina a hijos e hijas, así como $\mathrm{X}^{2}$ para observar diferencias entre los estilos parentales de los padres y las madres.

\section{Resultados}

La tabla 1 muestra cómo se perciben los papás a sí mismos con respecto a sus estilos de crianza. La mayoría de ellos se percibieron como buenos (25.4\%), comprensivos $(4.0 \%)$, responsables (8.5\%), cariñosos (5.0\%), y tolerantes (12.9\%). Por otro lado, un $22.9 \%$ se consideró como estricto y un $3.5 \%$ como consentidor.

La tabla 2 muestra cómo los padres percibieron a otros padres en sus prácticas de crianza, la mayoría los apreció como buenos (10.4\%), estrictos (8.0\%), y blandos (7.5\%). La generalidad indicó que no sabía cómo eran. Los padres se consideraron a ellos mismos con características de autoritativos (57.7\%). Se observaron diferencias significativas sobre su percepción acerca de ellos y los otros, t [191] =7.6, p<.00. Sin embargo, no se presentaron diferencias significativas entre cómo consideran los estilos de crianza las madres y los padres, $\mathrm{X} 2(3, \mathrm{~N}=155)$ $=4.38, p=.22$.

La tabla 3 muestra los resultados sobre la percepción que los padres tienen acerca de los modos de educar a sus hijos. Ellos indicaron

\section{Tabla 1.}

Frecuencias de la percepción de los estilos de crianza

\begin{tabular}{lll}
\hline Variable & Frecuencia & Porcentaje \\
\hline No sé & 14 & 7.0 \\
Autoritativo & 116 & 57.7 \\
Autoritario & 54 & 26.9 \\
Permisivo & 13 & 6.5 \\
Perdidos & 4 & 2.0 \\
Total & 201 & 100.0 \\
\hline
\end{tabular}

Tabla 2.

Frecuencia de la percepción del estilo de crianza de otros padres

\begin{tabular}{lll}
\hline Variable & Frecuencia & Porcentaje \\
\hline No sé & 122 & 60.7 \\
Autoritativo & 33 & 16.4 \\
Autoritario & 18 & 9.0 \\
Permisivo & 20 & 10.0 \\
Valores perdidos & 8 & 4.0 \\
Total & 201 & 100.0 \\
\hline
\end{tabular}


que mandarlos a la escuela (31.3\%), enseñar valores (16.9\%), y comunicarse (15.4) es lo más importante que pueden hacer para educarlos.

La tabla 4 muestra las formas cómo los padres reportaron que educan a sus hijas. Al igual que sus hijos piensan que lo más importante es mandarlas a la escuela (14.4\%), comunicarse (11.9\%) y enseñarles valores (11.9\%).

Como puede observarse en las dos tablas anteriores, resultaron muchos valores perdidos; parece ser que los padres no sabían qué contestar sobre cómo educar a sus hijos e hijas. Al comparar medias, no se observaron diferencias significativas, $t[54]=.18, \quad p .=85$, entre las formas de pensar de los padres sobre la educación de sus hijos e hijas. Igualmente, no resultaron diferencias significativas entre cómo padres y madres perciben que educan a sus hijos, $\mathrm{X} 2(6, \mathrm{~N}=155)=3.2, \mathrm{p}=.22$, e hijas, $\mathrm{X} 2$ (7, $\mathrm{N}=96)=4.9, \mathrm{p}=.66$.

La tabla 5 indica cómo los padres muestran afecto a sus hijos; el cariño verbal (33.8\%) es el que tuvo mayor porcentaje.

La tabla 6 muestra las formas en que los padres expresan afecto a las hijas: el afecto verbal $(28.9 \%)$ es el más frecuente, seguido de cariño instrumental (11.4\%). No existieron diferencias significativas de muestras de afecto a las hijas y los hijos, $t[59]=1.52, p=.13$. De la misma mane-

\section{Tabla 3.}

Frecuencias sobre las formas cómo los padres educan a los hijos

\begin{tabular}{lll}
\hline Variable & Frecuencia & Porcentaje \\
\hline Mandar a escuela & 63 & 31.3 \\
Enseñar buenos modales & 12 & 6.0 \\
Comunicarse & 31 & 15.4 \\
Ayudar en la escuela & 8 & 4.0 \\
Enseñar valores & 34 & 16.9 \\
Apoyarlos ideas y proyectos & 6 & 3.0 \\
Valores perdidos & 46 & 22.9 \\
Total & 201 & 100.0 \\
\hline
\end{tabular}

\section{Tabla 4.}

Frecuencias sobre las formas cómo los padres educan a las hijas

\begin{tabular}{lll}
\hline Variable & Frecuencia & Porcentaje \\
\hline Mandar a escuela & 29 & 14.4 \\
Buenos modales & 9 & 4.5 \\
Comunicarse & 24 & 11.9 \\
Ayudar en la escuela & 4 & 2.0 \\
Enseñar valores & 24 & 11.9 \\
Apoyarlos ideas y proyectos & 4 & 2.0 \\
Religión & 1 & .5 \\
Valores perdidos & 105 & 52.2 \\
Total & 201 & 100.0
\end{tabular}


Tabla 5.

Frecuencias sobre las formas de mostrar afecto a su hijo

\begin{tabular}{lll}
\hline Variable & Frecuencia & Porcentaje \\
\hline Cariño verbal & 68 & 33.8 \\
Cariño instrumental & 36 & 17.9 \\
Mostrar interés actividades & 28 & 13.9 \\
Reprender castigar & 3 & 1.5 \\
Educar valores & 1 & .5 \\
Valores perdidos & 48 & 23.9 \\
Total & 201 & 100.0 \\
\hline
\end{tabular}

Tabla 6.

Frecuencias sobre las formas de mostrar afecto a su hija

\begin{tabular}{lll}
\hline Variable & Frecuencia & Porcentaje \\
\hline Cariño verbal & 58 & 28.9 \\
Cariño instrumental & 23 & 11.4 \\
Interés actividades & 14 & 7.0 \\
Educar valores & 2 & 1.0 \\
Valores perdidos & 100 & 49.8 \\
Total & 201 & 100.0 \\
\hline
\end{tabular}

\section{Tabla 7.}

Frecuencias sobre las formas que los padres utilizan para disciplinar

\begin{tabular}{lll}
\hline Variable & Frecuencia & Porcentaje \\
\hline Nada & 11 & 5.5 \\
Castigo físico & 1 & .5 \\
Castigo verbal & 31 & 15.4 \\
Aconsejar y platicar & 52 & 25.9 \\
Quitarle cosas que le gustan & 55 & 27.4 \\
Valores perdidos & 49 & 24.4 \\
Total & 201 & 100.0 \\
\hline
\end{tabular}

ra, no se encontraron diferencias significativas entre cómo se perciben las madres y los padres en la muestras de efecto a sus hijos, X2 (6, $\mathrm{N}=153)=9.4, \mathrm{p}=.15 \mathrm{y}$ a sus hijas, $\mathrm{X} 2(4, \mathrm{~N}=101)$ $=3.3, p=.49$.

La tabla 7 muestra las formas que los padres utilizan para disciplinar a sus hijos; la que más se reportó fue quitarles cosas que les gustan $(27.4 \%)$ y aconsejar y platicar $(25.9 \%)$.

La tabla 8 muestra las formas de disciplinar a la hija cuando no obedece. Los padres indicaron que aconsejar y platicar (17.9\%) es lo que más utilizan con sus hijas, seguido por el castigo verbal (15.4\%). 
Tabla 8.

Frecuencias sobre las formas que los padres recurren para disciplinar

\begin{tabular}{lll}
\hline Variable & Frecuencia & Porcentaje \\
\hline Nada & 7 & 3.5 \\
Castigo físico & 2 & 1.0 \\
Castigo verbal & 31 & 15.4 \\
Aconsejar platicar & 36 & 17.9 \\
Quitarle cosas que le gustan & 19 & 9.5 \\
Valores perdidos & 103 & 51.2 \\
Total & 201 & 100.0 \\
\hline
\end{tabular}

No se mostraron diferencias significativas entre lo que los padres indicaron sobre cómo disciplinan a las hijas y los hijos (t $[60]=1.5$, $\mathrm{p}=.13$ ). Asimismo, no existieron diferencias entre cómo se perciben las madres y los padres en la disciplina de su hijo, $\mathrm{X} 2 \quad(6, \mathrm{~N}=152)=6.7$, $\mathrm{p}=.34$, e hija, $\mathrm{X} 2(6, \mathrm{~N}=98)=5.3, \mathrm{p}=.49$.

\section{Discusión}

Los resultados nos muestran que los padres tienen una buena percepción de ellos mismos, la mayoría se consideró como autoritativo y piensan que son buenos con sus hijos y que tienen una relación amistosa y razonable con ellos. Además, indicaron que existe una participación democrática entre los miembros de la familia y que los padres se involucraron en las actividades de sus hijos. Un porcentaje muy bajo se consideró como estricto, con estrategias punitivas, o que utiliza el castigo, es decir, aplicar el estilo parental autoritario según la clasificación de Baumrind (1983). Una cantidad mínima de padres y madres se consideró como consentidores, denominados por Baumrind (1983) como permisivos.

Por otro lado, la mayoría de los padres y las madres, porque no se presentaron diferencias significativas entre ellos, expresaron que la me- jor manera de educar a sus hijos es mandarlos a la escuela. Esto nos indica que la educación fue percibida como la forma escolarizada de introducir la formación, las habilidades, las destrezas y conocimientos que necesitan sus hijos para desarrollarse. La enseñanza de valores y la comunicación de ellos hacia sus hijos quedaron en segundo plano. Sin embargo, como lo indica Bradley (2002), los niños necesitan la estimulación de los padres, es decir, relacionarse con objetos, eventos, experiencias, condiciones y situaciones dentro de la familia, que les puedan ayudar a su desarrollo cognitivo, psicológico y social. Es muy importante que los padres y madres se involucren en dicho proceso educativo y que no lo determinen como una enseñanza escolarizada, ya que son ellos los que ayudan a modular las emociones para su ajuste con las demandas ambientales.

Los padres y las madres demuestran su cariño a las hijas y los hijos de manera verbal; ellos se percibieron como afectuosos con sus hijos e hijas; las relaciones amorosas con los hijos ayudan a su ajuste emocional. El desarrollo de los niños es mejor cuando viven en ambientes responsivos: los requerimientos de ayuda son proporcionados a tiempo y de manera predecible y satisfactoria (Bradley, 2002). Los ambientes aversivos y castigantes reducen el desarrollo emocional y cognitivo del niño (Dishion \& McMahon, 1998). El mostrar 
interés en las actividades de sus hijos también fue considerado como una muestra de afecto; esto es parte del estilo autoritativo y tiene que ver con el involucramiento de los padres en las actividades de sus hijos.

Los padres y las madres indicaron que la forma de disciplinar a sus hijos es quitándoles las cosas que les gustan, platicar con ellos y aplicar el castigo verbal. Es muy poco el castigo físico que reportaron. Lo anterior se pudiera deber a la deseabilidad social de la encuesta. Sin embargo, otros estudios de la región han mostrado que el castigo físico es muy común entre padres y madres de la región (Frías \& McCloskey, 1998). Las formas cómo los padres y madres revelaron se relacionan con sus hijos e hijas son muy positivas, por lo menos ellos lo percibieron así, y nos indicaron que están buscando la manera más positiva de que sus hijos e hijas se adapten de la mejor manera a su ambiente y puedan ser adultos exitosos. No se encontraron diferencias significativas sobre cómo ser perciben las madres y los padres a sí mismos ni diferencias en el trato con sus hijos e hijas.

Las limitaciones del estudio se refieren a la falta de respuestas de los padres, circunstancia que origina una cantidad de números perdidos que pudieran sesgar los datos. Por otro lado, evitaron hacer referencia a las prácticas punitivas de crianza, al reportar únicamente lo positivo; la negación del uso del castigo físico pudiera ser parte de esa búsqueda y lo entienden como una forma positiva de relacionarse con sus hijos. A pesar de tales problemas, este estudio nos proporciona un panorama sobre la percepción que tienen los padres sobre sus prácticas de crianza.
Alegre, A. (2011). Parenting Styles and Children's Emotional Intelligence: What do We Know? The Family Journal: Counseling and Therapy for Couples and Families, 19(1), 56-62.

Baptiste, D. R., Tolou-Shams, M., Miller, S. R., Mcbride, C. K., \& Paikoff, R. L. (2007). Determinants of parental monitoring and preadolescent sexual risk situations among African American families living in urban public housing. Journal of Child and Family Studies, 16, 261-274.

Baumrind, D. (1983). Rejoinder to Lewis's reinterpretation of parental firm control effects: Are authoritative families really harmonious? Psychological Bulletin, 94, 132-142.

Belsky, J. (2000). Conditional and alternative reproductive strategies: Individual differences in susceptibility to rearing experience. En J. Rodgers \& D. Rowe (Eds.), Genetic influences on fertility and sexuality (pp. 127-146). Boston, MA: Kluwer Academic.

Belsky, J., Putnam, S., \& Crnic, K. (1997). Coparenting, parenting, and early emotional development. New Directions in Child Development, $74,45-56$.

Bjorklund, D. F., Yunger, J.L., \& Pellegrini, A.D. (2002). The Evolution of Parenting and Evolutionary Approaches to Childrearing. En M. H. Bornstein, (Ed.), Handbook of Parenting, Second Edition, Volume 2, Biology and Ecology of Parenting (pp. 2-30). Mahwah, NJ.: Lawrence Erlbaum Associates, Publishers.

Bradley, R. H., Corwyn, R. F., McAdoo, H. P., \& García Coll, C.T. (2001). The Home Environments of Children in the United States Part I: Variations by Age, Ethnicity, and Poverty Status. Child Development, 72(6), 1844-1867.

Bradley, R. H. (2002). Environment and Parenting. En M. H. Bornstein, (Ed.), Handbook of Parenting, Second Edition, Volume 3, Social Ecology of Parenting (pp. 281-314). Mahwah, NJ.: Lawrence Erlbaum Associates, Publishers.

Carlo, G., Mestre, M. C., Samper, P., Tur, A., \& Armenta, B.E. (2010). The Longitudinal relations among dimentions of parenting styles, sympaty, prosocial moral reasoning, and prosocial behaviors. International Journal of Behavioral Development, 35(2), 116-124.

Cole, P., \& Tamang, B. L. (1998). Nepali children's ideas about emotional displays in hypothetical challenges. Developmental Psychology, 34, 640-646.

Collins, W. A., Maccoby, E. E., Steinberg, L., Hetherington, E. M., \& Bornstein, M. H. (2000). Contemporary research on parenting: The case for nature and nurture. American Psychologist, 55, 218-232.

Collins, K., \& Barber, H. (2005). Female athletes' perceptions of parental influences. Journal of Sport Behavior, 28, 295-314.

De Clercq, B., Van Leeuwen, K., De Fruyt, F., Van Hiel, A., \& Mervielde, I. (2008). Maladaptive personality traits and psychopathology in childhood and adolescence: The moderating effect of parenting. Journal of Personality, 76, 357-383.

Dishion, T. J., \& McMahon, R. J. (1998). Parental monitoring and the prevention of adolescent problem behavior: A conceptual and empirical formulation. Clinical Child and Family Psychology Review, 1, 61-75.

Dornbusch, S. M., Ritter, P. L., Leiderman, P. H., Roberts, D. F., \& Fraleigh, M. J. (1987). The relation of parenting style to adolescent school performance. Child Development, 58, 1244-1257.

Eiden, R. D., Edwards, E. P., \& Leonard, K. E. (2007). A conceptual model for the development of externalizing behavior problems among kindergarten children of alcoholic families: Role of parenting and children's self-regulation. Developmental Psychology, 43, 1187-1201.

Frías, M., y McCloskey, L. (1998). Determinants of harsh parenting in Mexico. Journal of Abnormal Child Psychology, 26,129-139.

Harkness, S. (2002). Culture and Parenting. En M. H. Bornstein, (Ed.), Handbook of Parenting, Second Edition, Volume 3, Social Ecology of Parenting (pp. 253-280). Mahwah, NJ.: Lawrence Erlbaum Associates, Publishers.

Hoff, E., Laursen, B., \& Tardif, T. (2002). Socioeconomic Status and Parenting. En M. H. Bornstein, (Ed.), Handbook of Parenting, Second Edition, Volume 3, Social Ecology of Parenting (pp. 231252). Mahwah, NJ.: Lawrence Erlbaum Associates, Publishers. 
Johnson, J. G., Cohen, P., Chen, H., Kasen, S., \& Brook, J. S. (2006) Parenting behaviors associated with risk for offspring personality disorder during adulthood. Archives of General Psychiatry, 63, 579587.

Khaleque, A., Rohner, R. P., \& Riaz, M. (2007). Perceived parental acceptance-rejection and psychological adjustment of children: A cross-cultural study in Finland, Pakistan, and the United States. Psychological Studies, 52, 114-119.

Knafo, A., \& Plomin, R. (2006). Parental discipline and affection and children's prosocial behavior: Genetic and environmental links. Journal of Personality and Social Psychology, 90, 147-164.

Larsson, H., Viding, E., Rijsdijk, F., \& Plomin, R. (2008). Relationships between parental negativity and childhood antisocial behavior over time: A bidirectional effects model in a longitudinal genetically informative design. Journal of Abnormal Child Psychology, 36, 633645.

Lengua, L. J. (2006). Growth in temperament and parenting as predictors of adjustment during children's transition to adolescence. Developmental Psychology, 42, 819-832.

LeVine, R. A., Dixon, S., LeVine, S., Richman, A., Leiderman, P. H., Keefer, C. H., \& Brazelton, T. B. (1994). Child care and culture: Lessons from Africa. Cambridge, England: Cambridge University Press.

Luyckx, K., Tildesley, E. A., Soenens, B., Andrews, J.A., Hampson, S.E., Peterson, M., \& Duriez, B. (2011). Parenting and Trajectories of Children's Maladaptive Behaviors: A 12-Year Prospective Community Study. Journal of Clinical Child \& Adolescent Psychology, 40(3), 468-478.

Maccoby, E. E., \& Martin, J. A. (1983). Socialization in the context of the family: parent-child interaction. En P. H. Mussen (Series Ed.) y E. M. Hetherington (Vol. Ed.), Handbook of child psychology: Vol. 4. Socialization, personality, and social development (4th ed., pp. 1-101) New York: Wiley.

Meunier, J.C., Roskam, I. \& Browne, D.T. (2010). Relations between parenting and child behavior: Exploring the child's personality and parental self-efficacy as third variables. International Journal of Behavioral Development, 35(3) 246-259.

Mogro-Wilson, C. (2008). The influence of parental warmth and control on Latino adolescent alcohol use. Hispanic Journal of Behavioral Sciences, 30, 89-105.

Morris, A. S., Silk, J. S., Steinberg, L., Myers, S. S., \& Robinson, L. R (2007). The role of the family context in the development of emotion regulation. Social Development, 16, 361-388.

Nord, C. W., Lennon, J., Liu, B., \& Chandler, K. (2000). Home literacy activities and signs of children's emerging literacy: 1993 and 1999. Education Statistics Quarterly, 2, 19-27.

Pan, B. A., Rowe, M. L., Singer, J., \& Snow, C. E. (2005). Maternal correlates of toddler vocabulary production in low-income families. Child Development, 76, 763-782.

Raikes, H., Pan, B. A., Luze, G., Tamis-LeMonda, C. S., Brooks-Gunn, J., Constantine, J., et al. (2006). Mother-child book reading in lowincome families: Correlates and outcomes during the first three years of life. Child Development, 77, 924-953.

Rodríguez, E. T., Tamis-LeMonda, C. S., Spellmann, M. E., Pan, B. A., Raikes, H., Lugo-Gil, J., et al. (2009). The formative role of home literacy experiences across the first three years of life in children from low-income families. Journal of Applied Developmental Psychology, 30, 677-694.

Sanders, M. (2008). Triple P-Positive Parenting Program as a public health approach to strengthening parenting. Journal of Family Psychology, 22, 506-517.

Suldo, S. M., \& Huebner, E. S. (2004). The role of life satisfaction in the relationship between authoritative parenting dimensions and adolescent problem behavior. Social Indicators Research, 66, 165195.

Townsend, N. W. (2000). Male fertility as a lifetime of relationships: Contextualizing men's biological reproduction in Botswana. En C.
Bledsoe, S. Lerner, \& J. I. Guyer (Eds.), Fertility and the male lifecycle in the era of fertility decline (pp. 343-364). New York: Oxford University Press.

Van Leeuwen, K. G., \& Vermulst, A. A. (2004). Ghent Parental Behavior Questionnaire: Psychometric properties; parents ratings; factor structure; internal consistency. European Journal of Psychological Assessment, 4, 283-298.

Wachs, T. D. (2000). Necessary but not sufficient. Washington, DC: American Psychological Association.

Wang, Q., Pomerantz, E. M., \& Chen, H. (2007). The role of parents control in early adolescents' psychological functioning: A longitudinal investigation in the United States and China. Child Development, 78, 1592-1610 